\title{
Oral care of elderly patients: nurses' knowledge and views
}

\author{
A J Preston, S Punekar, M A Gosney
}

\begin{abstract}
Summary
It is important that healthcare professionals caring for the elderly in hospitals have a core knowledge of the orodental care requirements of their patients. The aim of this study was to determine the knowledge and views of nurses working on acute and rehabilitation care of the elderly wards about orodental care. One hundred nurses and healthcare assistants took part in this questionnaire study of which 58 were qualified nurses and 70 had been employed on care of the elderly wards for two or more years. Although the majority of the respondents were registered with a dentist and attended regularly, 40 did have 'some anxiety' about visiting their dentist. Approximately half of the study population regularly gave advice to their patients about dental care but their knowledge of and reasons for providing oral care and advice was often incorrect. The group's understanding of the availability of dental treatment provided by the National Health Service was also often inaccurate. It was concluded that a better core knowledge of the orodental care of older patients is required by all healthcare professionals who care for this group. It is also important that individuals in whom anxiety is associated with their own dental experience do not neglect to give orodental health advice to their patients.
\end{abstract}

Keywords: oral care; elderly; nurses' knowledge

We have previously highlighted that the orodental status of many patients admitted to acute care of the elderly and rehabilitation wards is poor. ${ }^{1}$ Many edentulous patients are lacking dentures and a large proportion of elderly dentate individuals requires periodontal (gum) treatment, dental extractions and restorations (fillings). ${ }^{2}$ Conditions such as oral candidiasis, denture stomatitis, denture-irritation hyperplasia, and traumatic ulceration may also occur as a result of oral neglect. In addition, older people are less inclined to complain about oral or dental conditions unless they are in pain and therefore much oral pathology remains both undiagnosed and untreated.

Admission to hospital is not only a time for the active management of the presenting disease but also an excellent opportunity for health promotion and screening for undetected pathology. This is a responsibility of all health- care professionals caring for older patients. Nurses should perform daily oral care for many older people on acute, subacute and rehabilitation wards; however, much of their own education in this important area has been provided during their early training and regular updates may not occur. ${ }^{3}$ Additionally, many people, including healthcare professionals, have anxieties about their own dental attendance and treatment, which may affect the oral care advice that they give.

We therefore surveyed a group of nurses and healthcare assistants working on care of the elderly wards to determine whether their own experiences and views on dental care influenced the advice and care that they gave to older patients. We also ascertained whether the nurses' understanding of some aspects of basic oral health care was correct.

\section{Materials and methods}

Initially, 115 nurses and healthcare professionals working regularly on care of the elderly wards were contacted and their informed consent sought to take part in the study; night staff were included as part of the daytime rotations. Participation was on a voluntary basis and the anonymity of the respondents' data was assured. The nurses were working in three areas: acute admission areas for care of the elderly, a subacute unit to which patients are either admitted with less severe illnesses or are transferred during a period of hospitalisation, and thirdly, designated rehabilitation wards.

The questionnaire was designed to determine the subjects' experience of and attitude towards their own orodental care, their knowledge of oral disease, and the nature and extent of the oral-care advice that they give to their elderly patients. Percentages for the subjects' responses were calculated. Statistical analysis for comparing subgroups' responses was analysed using a Chi-square test (statistical significance at $\mathrm{p}<0.05)$.

\section{Results}

There was an $87 \%$ response rate with 100 nurses and healthcare professionals completing the questionnaire. Of these, 58 were qualified nurses, 29 were healthcare assistants and the remaining 13 were a variety of 'agency' and student nurses. Just less than half of the respondents were employed on a subacute 
Table Summary of total group responses $(n=100)$

\begin{tabular}{lll}
\hline & Yes (\%) & No (\%) \\
\hline Are you registered with a dentist ? & 86 & 14 \\
Are you anxious about dental attendance ? & 40 & 60 \\
Do you give oral care advice to the elderly in your care? & 43 & 57 \\
Is it important for edentulous individuals to see a dentist regularly? & 82 & 18 \\
Should dentures be removed at night? & 83 & 17 \\
Are dentures 'free' to all pensioners on the NHS? & 85 & 15 \\
\hline
\end{tabular}

ward (44); the remainder were divided between the rehabilitation unit (32) and acute wards (24).

The total 100 respondents were treated as a group with no differentiation between their qualifications and/or site of working. All were involved in the day-to-day oral care of patients in care of the elderly wards and their beliefs and opinions were therefore considered to be equally important.

The main results are summarised in the table. Although the majority of the staff were registered with a dentist, 14 were not and 40 had anxieties about attending the dentist. Of the 14 members of staff who were not registered with a dentist, $57 \%$ expressed dental anxiety, in contrast with those registered with a dentist in whom only $37 \%$ were anxious. A third of the total group (34\%) attended the dentist only when they had a dental problem, compared with the subgroup who had anxieties about dental attendance in which $70 \%$ visited the dentist only when they had a problem $(\mathrm{p}<0.01$, Chi-square). Thus, it was more likely that the anxious were less likely to routinely attend a dentist than those with no anxieties.

The percentage of nurses registered with a dentist in each of the three areas was similar; anxiety was reported, however, more commonly in staff working on the acute ward than on the subacute ward and least in those working on the rehabilitation ward ( $54 \%$ vs $36 \%$ vs $34 \%)$.

Half the sample attended their dentist every 6 months and a further 14 attended at yearly intervals. Interestingly, 79 of the group stated that dental visits should take place every 6 months although not all of this group practised this themselves. Two of the staff stated that they never attend the dentist.

In contrast to the 74 respondents who thought that the oral and dental health of older people was 'extremely important', only 54 considered that their own oral health was of such importance. Twelve nurses considered that their oral health was only 'moderately important', and of these the majority (67\%) were 'very anxious' about treatment.

Most (96\%) respondents stated that dentate elderly people should regularly attend the dentist. It is important to note, however, that only $82 \%$ believed that edentulous elderly people should regularly attend the dentist. Sixty-two per cent of the sample thought that dentures should be cleaned twice a day, while $11 \%$ thought that once-daily denture cleaning was sufficient.
Seventeen per cent of the staff considered that dentures did not need to be removed at night; the commonest reason given for the need for nocturnal denture removal was to prevent choking. Forty-three per cent of the staff regularly gave advice to older patients about their dental care and in the majority of cases the advice given pertained to the use of denture cleaning agents. Of the 57 nurses who gave no regular advice to older patients, a similar proportion were employed in the subacute and rehabilitation units $(61 \%$ and $63 \%$ of the total nurses, respectively), whilst $46 \%$ of nurses working in the acute unit failed to give advice to their patients (Chi-square, $\mathrm{p}=\mathrm{ns}$ ).

There was a difference between site of employment with regard to the nurses' views on the removal of dentures. Only $4 \%$ of the acute ward nurses said that dentures should not be removed in contrast to $25 \%$ of the subacute nurses and $16 \%$ of those nurses working on the rehabilitation unit (Chi-square, $p=n s$ ).

The pathological conditions other than dental caries (tooth decay) and periodontal disease (gum disease) that were known by the respondents to affect the mouth included oral 'thrush' $(47 \%)$ and 'dry mouth' (20\%). It is noteworthy that only $22 \%$ of the staff knew of the possibility of cancer occurring in the oral cavity.

Most nurses (85\%) believed that elderly people could obtain dentures free on the NHS, although those who realised that dentures are not free correctly estimated the cost of a set of complete dentures to be between $£ 100$ and $£ 200$. The 40 nurses who were anxious about dental treatment were more likely to be incorrect in their knowledge of oral healthcare especially about the non-availability of free dentures on the NHS for the elderly, compared with the 'non-anxious' group (Chi-square, $\mathrm{p}=0.02$ ).

\section{Discussion}

The results of our study broadly agree with recent similar studies ${ }^{4-6}$ in so much as deficiencies exist in healthcare professionals' knowledge of the appropriate oral care regimens for elderly patients. We have also shown that the deficiencies in their knowledge appear to be associated with their own anxiety about visiting the dentist. Additionally, nurses' practices with regard to regular dental attendance do not always correlate with their views on how often one should attend a dentist.

The healthcare professionals who volunteered to participate in the study exhibited more regular dental attendance and less anxiety than that found in the general population. Sixty-four per cent of the nurses were regular dental attenders and $40 \%$ were anxious about dental treatment, compared with $39 \%$ and $56 \%$, respectively, in the general population. ${ }^{7}$ Thus, more regular attenders may be influenced by the contact with healthcare issues and also the proximity of a university dental hospital. It does not, however, explain why the group as a whole was less anxious but may suggest relative underreporting in our study. 
Interestingly, the levels of anxiety about dental treatment and incorrect knowledge about orodental care were more prevalent in the members of staff working on the acute ward. The reason as to why the site of working should influence an individual's anxiety about dental attendance or understanding of and advice given about orodental care is not clear. It cannot, however, be explained by the nurses' levels of experience, since these were similar in each site. Previous studies of similar groups of nurses, however, have found that the mean age on subacute wards to be statistically significantly higher. ${ }^{8}$

The importance of regular dental attendance is clear, as dental practitioners not only screen patients for dental decay and periodontal disease, but also for a plethora of oral soft tissue infections, ${ }^{9}$ tumours and other abnormalities. ${ }^{10}$ Hence, even edentulous patients should be examined by a dentist every 6 months. This important point was not realised by nearly a fifth $(18 \%)$ of the respondents. It should, however, be noted that nurses working in acute wards would, by definition, have less opportunity to address patients' oral needs than nurses working on the subacute wards, due to the nature of acute care. Additionally, there are groups who are at particular risk of conditions such as denture stomatitis and oral thrush because of the presence of complete dentures, a common finding in studies of this population in Merseyside. ${ }^{1}$

Although the retention of the natural dentition is increasing into old age ${ }^{7}$ with the concomitant problems of root caries, toothwear and periodontal disease, many elderly patients still have dentures. The knowledge, however, of healthcare professionals about denture care was lacking in some instances. Most of the staff correctly stated that dentures should be regularly cleaned, but $17 \%$ of the staff did not realise that dentures should always be removed at night. Those who did realise the importance of nocturnal denture removal attributed the reason for doing so to the 'prevention of choking'. This reason is not viewed by the dental profession as a significant cause for concern; rather dentures should be removed at night to prevent such conditions as denture stomatitis and acute pseudomembranous candidiasis (thrush). These conditions are relatively common; approximately $30 \%$ of denture wearers have some form of candidal infection, ${ }^{9}$ whilst in a study of 465 Danish eld-

1 McNally L, Gosney MA, Field EA, Doherty U. The denta status of elderly in-patients. Age Aging 1998;27:47.

2 Steele JG, Walls AWG, Ayatollahi SMT, Murray JJ. Majo clinical findings from a dental survey of elderly people in three different English communities. Br Dent $\mathscr{f}$ 1996;180 $17-23$.

3 Longhurst RH. A cross-sectional study of the oral healthcare instructions given to nurses during their basic training. Br Dent $\mathcal{F}$ 1998; 184:453-7.

4 Miller R, Rubinstein L. Oral health care for hospitalised patients: the nurse's role. 7 Nurs Educ 1987;26:362-6.

5 Logan HL, Ettinger R, McLeran H, Casko R. Common Logan HL, Ettinger R, McLeran H, Casko R. Common
misconceptions about oral health in the older adult: nursing practices. Spec Care Dent 1991;11:213-24.

6 Wardh I, Andersson L, Sorenson S. Staff attitudes to oral health care. A comparative study of registered nurses, nursing assistants and home care aides. Gerodontology 1997;14 28-32.

\section{Summary points}

- nurses' knowledge of and attitude towards their own orodental care may influence the oral care advice that they give to elderly patients

- nurses' knowledge of oral health issues was sometimes incorrect; in some cases, they were potentially giving inappropriate advice regarding oral care to the patients for whom they were caring

- a core knowledge about the orodental care of older patients is required by all healthcare professionals who care for this group

erly, Budtz-Jorgensen et al ${ }^{11}$ determined that the prevalence of denture stomatitis was $65 \%$; the prevalence of acute candidal infections is generally much lower. Indeed, in $43 \%$ of the population surveyed, oral candidal infection has been shown to be present. ${ }^{12}$

Not surprisingly, the vast majority $(85 \%)$ of the staff held the common misconception that dentures are provided free to pensioners on the NHS. This is not the case, unless the patient receives income support. The state pension is not, in itself, a pre-requisite for free denture provision. Those who did realise that dentures are not provided free correctly estimated that the cost of a complete set of dentures on the NHS is between $£ 100$ and $£ 200$ (the present

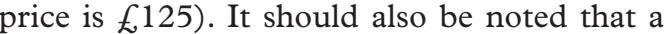
complete set of dentures obtained from a nonNHS dental practitioner often costs far more.

The main conclusion of this study, therefore, is that healthcare professionals caring for older patients require regular and contemporaneous updates on the oral care of the elderly. This observation agrees with those of Ridley ${ }^{13}$ and Longhurst. $^{3}$ There is a qualification, the Certificate in basic oral health promotion from the Royal Society of Health ${ }^{14}$ that healthcare professionals should be encouraged to pursue. In addition, Longhurst ${ }^{3}$ has outlined review papers on oral care for the elderly that are of particular benefit to nurses and healthcare professionals. The opportunity for educating any healthcare professional who has anxieties about their own dental care should not be lost, since this group is often more likely to be incorrect in their basic knowledge of orodental care.

7 Todd JE, Lader D. Adult dental health 1988 United Kindgom. London: HMSO, 1991

8 Maestri-Banks A, Gosney M. Nurses response to palliative care in a geriatric unit. Int $\mathcal{F}$ Palliative Care 1997;3:345-50. 9 Marsh PD, Martin MV. Oral microbiology, 3rd edn. London: Lea and Chapman, 1992.

10 Shafer WG, Hine MK, Levy BM. A textbook of oral pathology, 4th edn. London: WB Saunders Co, 1983.

11 Budtz-Jorgensen E, Stenderup A, Grabowski M. An epidemiological study of yeasts in elderly denture wearers. Comm Dent Oral Epidemiol 1975;3:115-9.

12 Preston AJ, Gosney MA, Noon S, Martin MV. Oral flora of elderly patients following acute medical admission. Gerontolelderly patients follo

13 Ridley KJ. Including the nurse in oral care. 7 Hosp Dent Pract 1980;14:158-9.

$1980 ; 14: 158-9$.
Royal Society of Health. Certificate in basic oral health promotion: syllabus and regulations. London: RSH, 1997. 